Original Article

\title{
Mahjong playing and eye-hand coordination in older adults-a cross-sectional study
}

\author{
William W.N. Tsang, PhD ${ }^{1)^{*}}$, Gloria C.K. Wong, MSc ${ }^{1)}$, Kelly L. GaO, MPT ${ }^{1)}$ \\ 1) Department of Rehabilitation Sciences, Hong Kong Polytechnic University: Hung Hom, Kowloon,
} Hong Kong, SAR, China

\begin{abstract}
Purpose] Eye-hand coordination declines with age, but physical activity is known to slow down the degeneration. Playing mahjong involves lots of eye-hand coordination. The objective was to investigate the relationship between playing mahjong and eye-hand coordination in older adults using a fast finger-pointing paradigm. [Subjects and Methods] Forty-one community dwelling older adults aged sixty or above were recruited by convenience sampling in this cross-sectional study. They were tested on their ability to point quickly and accurately 1) toward a stationary visual target and 2) toward a moving visual target. [Results] The mahjong players demonstrated significantly better end-point accuracy when pointing with their non-dominant hand toward a stationary target. They also demonstrated significantly faster movement of their dominant hands; shorter reaction times and better end-point accuracy when pointing with their non-dominant hands toward a moving target. [Conclusion] Mahjong players have better eye-hand coordination than non-players. Playing mahjong could usefully be introduced to older adults as a leisure time activity.

Key words: Aging, Eye-hand coordination, Mahjong
\end{abstract}

(This article was submitted May 27, 2016, and was accepted Jul. 8, 2016)

\section{INTRODUCTION}

Eye-hand coordination is essential for humans, as many activities in daily life require precise eye and hand functions. It is an intricate and complex function of the central nervous system ${ }^{1)}$. In general, eye-hand coordination involves sensory input from the tactile kinesthetic and proprioceptive systems as well as vision, as they provide the initial information needed for formulating motor plans and regulating the initiation and execution of movements. After a movement is initiated, feedback from the sensory and motor systems will modify other movements such as postural control that are to be incorporated into the eye-hand coordination activity ${ }^{2,3)}$.

As an important perceptual-motor function, eye-hand coordination is not spared from the effects of aging ${ }^{4}$. Different studies have shown that aging leads to a decline in different aspects of eye-hand coordination ${ }^{4,5}$.

Mahjong is one of the most popular entertainment games among Chinese. Older adults in particular enjoy playing mahjong because the game requires attention control and alertness, heightened speed and volume of information processing, visual-motor coordination and the game's gambling element is also attractive ${ }^{6}$. In addition, playing mahjong indirectly improves psychological well-being by way of social interaction ${ }^{7}$. Three aspects of eye-hand coordination are demanded in the play (please refer to Appendix 1. for a brief description of the game). The first is reaction time. A slow appreciation of the utility of discarded tiles decreases the chances of winning, as the following player may quickly take his/her turn to get the tile. Speed of movement is also important. Slow play is rare, as there is usually a subconscious rhythm to the play, although playing slower incurs no penalty. The last attribute demanded is accuracy. A penalty may be incurred if a player plays the wrong tile and violates the rules of play. There has been no previous research in the effects of playing mahjong on eye-hand coordination. Since the game is so traditional and popular, it would be interesting to understand its effects on eye-

*Corresponding author. William W.N. Tsang (E-mail: william.tsang@polyu.edu.hk)

(C2016 The Society of Physical Therapy Science. Published by IPEC Inc.

This is an open-access article distributed under the terms of the Creative Commons Attribution Non-Commercial No Derivatives (by-nc-nd) License $<$ http://creativecommons.org/licenses/by-nc-nd/4.0/>. 
hand coordination, particularly among older players. This study was therefore designed to investigate the effects of playing mahjong on the eye-hand coordination of older adults. Reaction time, movement time and the accuracy of fast finger-pointing while seated were all studied.

\section{SUBJECTS AND METHODS}

This was an exploratory study with a cross-sectional design, and a sample size of 40 was planned. The subjects were required to be aged 60 or older and independent in their activities of daily living. They had to be able to communicate and follow instructions. Those in the mahjong group had at least three years of experience playing mahjong at least three times per week for at least half an hour each time, or for a total of 1.5 hours per week. The subjects were recruited in Hong Kong where such devotion to the game is not difficult to find. In fact the group's experience playing mahjong ranged from 6-40 years (mean=26.7 years) and the frequency ranged from 3-35 hours/week (mean=12.1 hours/week). The control group was chosen to fit the same criteria except that they did not play mahjong. The recruitment period was from July 2013 to December 2013.

Exclusion criteria comprised those who were Tai Chi practitioners ${ }^{8)}$ or who regularly played sports involving a significant amount of eye-hand coordination (e.g. golf, badminton, tennis, etc.). Those with severe visual problems or any eye pathology (e.g. glaucoma or cataracts) were also excluded, except those whose vision was corrected sufficiently that they could pass a visual screening test. Those who had upper limb problems or lacked enough functional range of arm movement for testing were also excluded. Those who had some medical contraindication such as metastatic cancer or a recent fracture of the arm and subjects who had neurological or movement-related disorders were also excluded. Ethics approval was obtained from the Ethics Committee of the Hong Kong Polytechnic University. Interested subjects were invited to the screening tests. After thorough explanation of the study, written consent was signed.

Several screening tests were conducted, including 1) a general health questionnaire; 2) the Mini-Mental Status Examination (MMSE) $)^{9,10)}$; 3 ) a visual acuity test ${ }^{11)}$; 4 ) a test of the functional range of movement of the upper limbs; and 5) a modified physical activity level questionnaire ${ }^{12,13)}$.

Demographic data including age, gender, hand dominance and arm lengths were collected for each subject. The tests were conducted in sitting position with the height of the chair adjusted so that the subject's elbows, hips, knees and ankles were positioned at approximately $90^{\circ}$. He or she faced a visual display unit (VDU) of which the upper edge was adjusted to each subject's eye level. A mark was positioned in the middle of upper edge of the VDU, and the subjects were required to gaze at it when there was no signal on the screen.

Electromyographic (EMG) surface electrodes (B\&L Engineering Division of Pinsco Inc., Santa Ana, CA, USA) were placed on the anterior deltoid muscle (prime mover for arm reaching movement) of the tested arm with adhesive tape and electrode gel after the skin had been prepared using the sand paper and alcohol as recommended by Cram \& Kasman ${ }^{14)}$. A ground electrode was placed on the bony prominence of the elbow. EMG signals were recorded at a total gain of 320 times, a total input impedance of $>100$ MegaOhms, and with a bandwidth of $12-3,000 \mathrm{~Hz}$. The signals were sampled at $1,000 \mathrm{~Hz}$ and stored for off-line analysis using an analogue to digital conversion card (National Instruments NI DAQCard-6024E). The EMG signals were processed using the LabView software suite (National Instruments, Austin, TX, USA). The signals were full-wave rectified and smoothed using a second-order Butterworth low pass filter with a cut-off frequency of $10 \mathrm{~Hz}$.

The assessment procedures were first explained to the participants. Familiarization trials of each testing protocol were performed. For standardization, each subject's non-dominant arm was assessed after the dominant arm. The subjects were required to touch the visual target as quickly as possible after it appeared on the VDU. They were asked to sit in front of the VDU and both hands on fixed hand prints on the table. They were asked to sit upright with their backs touching the back of the chair to minimize any trunk movement during the assessment. For standardization, the hand prints were positioned so that it was $10 \mathrm{~cm}$ from the tips of both index fingers to the surface of the VDU. The subjects were asked to relax both arms before and after each pointing trial, and this was monitored using the EMG signals. The onset of muscle activity was identified as the point where the EMG signal fired and deviated more than 3 standard deviation from the baseline. The point was determined using a tailor-made LabView software program, but was checked visually. The EMG thresholds were preset during the subjects' resting periods. The visual target would not appear if their EMG signal was above the threshold. Participants were instructed to use the index finger pad of the hand being assessed to touch the visual signal as quickly and accurately as possible after it appeared on the VDU. During each trial, a 'beep' sound was generated by the computer (a preparatory signal) to alert the subject before the appearance of the visual signal. But a visual signal sometimes might not follow the beep sound to prevent subjects' anticipation. The visual signal was a black circle $1.2 \mathrm{~cm}$ in diameter. Each subject underwent testing with two objective measurement protocols: 1) fast finger-pointing toward a stationary visual signal, and 2) fast finger-pointing toward a moving visual signal.

For the first protocol, subjects were instructed to touch the visual target when it appeared on the screen. The target was stationary and in the middle of the VDU. The assessing hand returned to the starting position and relaxed after finishing each task, otherwise the next visual target would not appear. During the whole trial, the other hand remained resting on the handprint and relaxed. The visual target appeared 10 times.

For the second protocol, the visual signal moved from contralateral to ipsilateral with respect to the hand being assessed at a speed of $12 \mathrm{~cm} / \mathrm{s}$. Subjects were instructed to touch the moving target as quickly and as accurately as possible when it 
Table 1. The basic characteristics of mahjong players and non-players

\begin{tabular}{lcc}
\hline Demographic profiles & $\begin{array}{c}\text { Non-mahjong players } \\
(\mathrm{n}=20)\end{array}$ & $\begin{array}{c}\text { Mahjong players } \\
(\mathrm{n}=21)\end{array}$ \\
\hline Age (years) & $64.3 \pm 5.4$ & $65.9 \pm 6.7$ \\
Dominant arm length (cm) & $69.4 \pm 2.8$ & $69.4 \pm 4.9$ \\
Non-dominant arm length (cm) & $69.1 \pm 2.8$ & $69.1 \pm 4.7$ \\
Gender (M/F) & $6 / 14$ & $5 / 16$ \\
MMSE scores & $27.4 \pm 1.9$ & $28.3 \pm 1.9$ \\
Physical activity level & & \\
Light $<4$ METs (n) & 6 & 12 \\
Moderate 4-5.5 METs (n) & 10 & 2 \\
Heavy $>5.5$ METs (n) & 4 & \\
\hline Data are presented as the mean \pm SD & & \\
MMSE: the Mini-Mental Status Examination; MET: metabolic equivalent; SD: standard deviation
\end{tabular}

appeared on the screen. The hand returned to the starting position and relaxed after finishing each task, otherwise the next target would not appear. During the whole trial, the other hand remained over the hand print and relaxed. The target appeared 10 times.

Reaction time, movement time and end-point accuracy were measured during the fast finger-pointing tasks. Reaction time was defined as the interval between the target's appearance on the VDU and the onset of EMG response preceding muscle contraction. Movement time was the time from the onset of EMG response to touching the target on the VDU. End-point accuracy was defined as the absolute error between the subject's touch location and the center of the target.

In our laboratory, similar testing protocols have been adopted. The reliability on reaction time, movement and accuracy all showed moderate to very good test-retest repeatability $(\mathrm{ICC}=0.68-0.97)^{8}$.

For data analysis, independent t-tests were used to compare the ages, heights, arm lengths and MMSE scores between the two groups, and gender and physical activity levels were compared using chi-squared tests. Since the starting position of the hand with respect to the VDU was fixed for all participants, differences in arm length might constitute a covariate in the finger-pointing task. Arm length was thus treated as a covariate in the statistical analysis if a significant difference was found. Arm length was defined as the distance between a subject's acromion and the tip of the middle finger. Multivariate analysis of variance (MANOVA) was employed to compare the eye-hand coordination of each subject's dominant and non-dominant hands in each of the protocols. Post-hoc analysis using univariate tests was conducted for each of the outcome measures, namely reaction time, movement time and end-point accuracy. A significance level $(\alpha)$ of 0.05 was chosen as the threshold of statistical significance.

\section{RESULTS}

Forty one community-dwelling elderly persons were recruited by convenience sampling. They were divided into a mahjong playing group ( 6 men, 14 women; mean age \pm standard deviation, $64.3 \pm 5.4$ years) and a non-mahjong playing group (5 men, 16 women; 65.9 \pm 6.7 years). Demographic data describing all the subjects are shown in Table 1. All the subjects recruited were right hand dominant. All the subjects had the scores above 24 on the MMSE, suggesting that they had no cognitive impairment ${ }^{9,10)}$.

Independent t-tests showed that there were no significant differences in average age, arm length or MMSE score between the mahjong playing and non-playing groups. The $\chi^{2}$ tests found no significant difference between the two groups in terms of gender or average physical activity level (Table 1). So the mahjong playing and non-playing groups were considered comparable in all other respects.

For the protocol of pointing toward a stationary visual target, no statistically significant difference was found between the dominant-hand-performance of the two groups in MANOVA test $(F(1,39)=1.472, \mathrm{p}=0.238)$ and also in univariate tests in terms of reaction time $(\mathrm{F}(1,39)=1.802, \mathrm{p}=0.187)$, movement time $(\mathrm{F}(1,39)=0.815, \mathrm{p}=0.372)$ or end-point accuracy $(\mathrm{F}(1,39)=1.313, \mathrm{p}=0.259$; Table 2$)$. For the non-dominant hand, there was again no statistically significant difference found in MANOVA test $(\mathrm{F}(1,39)=2.284, \mathrm{p}=0.095)$. Subsequent univariate tests showed no significant difference in reaction time $(\mathrm{F}(1,39)=0.098, \mathrm{p}=0.756)$ or movement time $(\mathrm{F}(1,39)=0.896, \mathrm{p}=0.350)$, but a significant difference was detected in the endpoint accuracy of the two groups $((\mathrm{F}(1,39)=4.949, \mathrm{p}=0.032$; Table 2$)$.

For the protocol of pointing toward a moving visual target, no statistically significant difference was found between the dominant-hand-performance of two groups in the MANOVA tests $(\mathrm{F}(1,39)=1.827, \mathrm{p}=0.160)$ and also univariate tests in terms of reaction time $(\mathrm{F}(1,39)=0.619, \mathrm{p}=0.436)$ or end-point accuracy $(\mathrm{F}(1,39)=1.578, \mathrm{p}=0.217)$, but their movement time was significantly different $(\mathrm{F}(1,39)=4.454, \mathrm{p}=0.042$; Table 3$)$. For the non-dominant hand, statistically significant difference was 
Table 2. Results of outcomes for mahjong players and non-players in finger pointing toward a stationary visual target

\begin{tabular}{lcc}
\hline Outcome measures & $\begin{array}{c}\text { Non-mahjong } \\
\text { players } \\
(\mathrm{n}=20)\end{array}$ & $\begin{array}{c}\text { Mahjong } \\
\text { players } \\
(\mathrm{n}=21)\end{array}$ \\
\hline Dominant hands & & \\
EMG reaction time (ms) & $383.4 \pm 77.5$ & $357.7 \pm 39.5$ \\
EMG movement time (ms) & $611.1 \pm 193.5$ & $569.6 \pm 81.6$ \\
End-point accuracy (mm) & $8.5 \pm 5.2$ & $7.1 \pm 2.3$ \\
Non-dominant hands & & \\
EMG reaction time (ms) & $377.8 \pm 45.8$ & $381.9 \pm 37.3$ \\
EMG movement time (ms) & $639.5 \pm 179.2$ & $598.0 \pm 88.5$ \\
End-point accuracy $(\mathrm{mm})$ & $12.8 \pm 7.5$ & $8.9 \pm 2.8^{*}$ \\
\hline
\end{tabular}

*Significant difference between the mahjong players and nonplayers.

Data are presented as the mean \pm SD. EMG: Electromyography
Table 3. Results of outcomes for mahjong players and non-players in finger pointing toward a moving target

\begin{tabular}{lcc}
\hline Outcome measures & $\begin{array}{c}\text { Non-mahjong } \\
\text { players } \\
(\mathrm{n}=20)\end{array}$ & $\begin{array}{c}\text { Mahjong } \\
\text { players } \\
(\mathrm{n}=21)\end{array}$ \\
\hline Dominant hands & & \\
EMG reaction time (ms) & $347.5 \pm 65.4$ & $333.6 \pm 42.4$ \\
EMG movement time (ms) & $649.5 \pm 239.0$ & $529.9 \pm 63.3^{*}$ \\
End-point accuracy (mm) & $10.2 \pm 6.3$ & $8.2 \pm 3.3$ \\
Non-dominant hands & & \\
EMG reaction time (ms) & $381.6 \pm 73.6$ & $335.1 \pm 32.6^{*}$ \\
EMG movement time (ms) & $629.9 \pm 199.4$ & $617.6 \pm 59.8$ \\
End-point accuracy (mm) & $16.3 \pm 11.5$ & $10.3 \pm 2.8^{*}$ \\
\hline
\end{tabular}

*Significant differences between the mahjong players and nonplayers.

Data are presented as the mean $\pm \mathrm{SD}$. EMG: Electromyograpy

found in MANOVA test $(\mathrm{F}(1,39)=3.671, \mathrm{p}=0.022)$ and also in the univariate tests for reaction time $(\mathrm{F}(1,39)=6.334, \mathrm{p}=0.016)$ and end-point accuracy $(\mathrm{F}(1,39)=4.793, \mathrm{p}=0.035)$, but not for movement time $(\mathrm{F}(1,39)=0.067, \mathrm{p}=0.797$; Table 3$)$.

\section{DISCUSSION}

Many older adults play mahjong at social gatherings and in their leisure time ${ }^{6}$. Studies have shown that playing mahjong can directly or indirectly promote social interaction ${ }^{7}$. Such cognitive stimulation can lower the level of distress ${ }^{7}$. To the best of our knowledge, this is the first study investigating the effects of mahjong playing on eye-hand coordination in older adults.

For the first protocol (pointing toward a stationary target), mahjong players' reaction times, movement times and accuracy are on average slightly but not significantly better than those of non-players when testing on their dominant hands (Table 2). In these study protocols, the older subjects were tested using a stable starting position and responding to a simple command. Pointing toward a stationary visual target might not have been challenging enough to reveal any inter-group differences, especially for the dominant hand. Spirduso ${ }^{2}$ has suggested that anticipation is one of the compensatory strategies for loss of coordination. Older people could develop ways to anticipate movements so that when required, as in playing mahjong, their movements can be quick and efficient. In the stationary finger-pointing protocol, anticipation could not be eliminated even though a visual signal sometimes did not appear after the preparatory signal.

In our daily activities, accuracy is often important role in eye-hand coordination. An older adult can often manage a task more slowly, but poor accuracy in locating and handling objects is often not acceptable. The dominant hand is, by definition, usually chosen for precise movements requiring dexterity. The non-dominant hand would be used less often. Playing mahjong requires bilateral hand activity. The movements demand precise hand skills which include building up blocks of tiles, getting a tile from a particular block and claiming a tile from the table. Other than in forced bilateral hand movements, experienced players usually use the 'nearer' hand to manipulate tiles. This enrolls the participation of the non-dominant hand much more than many other activities.

A significant difference was found in the end-point accuracy of the non-dominant hands in pointing toward a stationary target $\left(\mathrm{p}=0.032\right.$; Table 2). In this connection, Boisseau, Scherzer and Cohen ${ }^{5)}$ found that for both younger (36.8 \pm 2.5 years) and older (64.9 \pm 3.0 years) subjects, speed and accuracy increased with practice, showing that both age groups were capable of improving eye-hand coordination. Those investigators proposed that older subjects became more efficient in using feedback and make more effective corrections. Williams ${ }^{3}$ in his review also concluded that aging individuals can profit from practice or experience in a wide variety of perceptual and motor tasks. On the other hand, some researchers have found that older people often trade speed for accuracy ${ }^{15}$, because they generally prefer better accuracy to faster movement ${ }^{2}$. That may explain why mahjong players have better accuracy in the finger-pointing task.

Playing mahjong might also enhance joint proprioception, which is essential for accurate finger pointing ${ }^{8}$. Certain exercises like Tai Chi which put great emphasis on exact joint positioning have been shown to help improve joint proprioception $^{16,17)}$. Playing mahjong requires the player to build the blocks of tiles in a proper and preset manner. The four players have to position their blocks in a square castle shape. All these movements also require joint proprioception. Although there has been no research on the effect of playing mahjong on joint proprioception, playing could enhance joint proprioception and thus improving accuracy in pointing toward a stationary visual target.

When comparing with a stationary target, pointing quickly toward a moving target is substantially more demanding. When required to catch a moving visual signal, older adults usually take more time for information processing ${ }^{18}$. The pointing test 
simulates getting a pong in mahjong (Appendix 1). When the player has 2 of a kind and the latest discard gives him a pong, he/she must quickly call "pong" and picked up the discarded piece before the following player has a chance to get another tile from the blocks. Usually, the discarded tile would have a short period of 'flight' on the table floor after being thrown. Players must pay constant attention to what the other players' discards were, locate the required target and take the tile quickly.

The tests in this study showed that mahjong players are on average significantly quicker in pointing to a moving target using their dominant hands $(\mathrm{p}=0.042$, Table 3$)$. This is probably explained by the practice acquired during mahjong play ${ }^{5,17)}$. Usually each mahjong session involves at least four rounds of four games; i.e. at least sixteen games. During each game, there would be quite a lot of repeated reaching and grasping movements in getting or throwing down tiles. A session requires about 4 hours, depending on the players' speed. But usually there is a hidden rhythm during play and slow timing is rare. Some experienced players can even finish a session within 2 hours. In addition, just like other mental games, each game is a new exercise presenting a completely different combination of stimuli for the players ${ }^{6}$. Repeated mahjong playing could therefore be expected to maintain or improve players' fast finger-pointing performance.

In playing mahjong, constant attention and alertness are important ${ }^{6}$. Each player must remember which tiles thrown by other three players might be useful for a pong or chee. Response preparation is a controlled attentional process (which relies on the integrity of the frontal cortex), but it becomes impaired with advancing age. Regular physical activity is associated with better performance on reaction time tasks ${ }^{19}$. As the players pay close attention in the game, it should develop their visual detection and target identification/location skills. Since these factors contribute to the reaction time of normal eye-hand coordination $^{3}$, it may explain the significantly faster average reaction time of mahjong players $(\mathrm{p}=0.016)$ using their nondominant hands in pointing toward the moving target.

In addition, the mahjong players showed significantly better end-point accuracy $(\mathrm{p}=0.035)$ with a moving target. Playing mahjong requires players to move both hands simultaneously in 'washing' the tiles, building blocks of tiles, showing their own set of tiles, etc. Repeating such complex movements may improve arm control, which could lead to a better end-point accuracy.

As has been mentioned before, practice plays an important role in the end-point accuracy of non-dominant hand move-

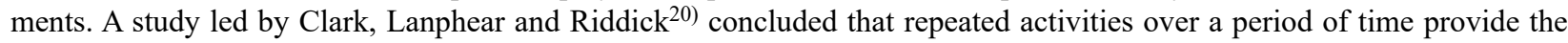
best results. That study documented the effect of video game playing on the motor responses of older adults (57-83 years). The subjects were asked to practice two video games two hours per week for 7 weeks. Both games involved making decisions about appropriate or needed changes in the speed and direction of arm and hand movements. The players in that study showed a six-fold improvement in their scores after the seven weeks. Mahjong players must similarly claim required tiles from the blocks or from the table repeatedly over a whole session. Such practice would be expected to lead to better end-point accuracy.

Several limitations were identified. First of all, simulating the eye-hand coordination movements of daily life is difficult, as they are complex. Playing mahjong involves not only simple eye-hand coordination, but also very complex mental work. A review by Spirduso $^{2)}$ has demonstrated that psychologists, motor behaviorists, and ergonomists are trying to use laboratory tasks to stimulate eye-hand coordination in daily physical activities but motor coordination is so complex that most researchers study either very simple movements or break complex skills into components and study each component in isolation. Furthermore, the need to reduce variability, achieve better accuracy, and place less stress on older subjects all lead to simpler movement protocols, as in the present investigation. Secondly, the present study was a cross-section in nature and a randomized clinical trial is required to explore any causal relationship between mahjong playing and speed and accuracy in finger-pointing tasks.

The small sample might also have affected the results. The average results of the mahjong players were generally better, but the differences did not achieve statistical significance.

Lastly, all the subjects recruited for this study were right-handed. The results cannot automatically be extended to lefthanded people. The hands are controlled by different hemispheres that are responsible for different functions ${ }^{21)}$.

In conclusion, the older mahjong players studied showed better average end-point accuracy when pointing with their non-dominant hand toward a stationary target. They also demonstrated significantly faster movement of the dominant hand, shorter reaction time and better end-point accuracy with the non-dominant hand in locating a moving visual target. Mahjong playing, a traditional and popular game in Chinese society, is easily accepted by older adults of disparate health status. With its advantages in nurturing social interaction and mental training, mahjong playing can be introduced to the elderly as a low cost leisure time activity requiring little space. Further studies using a randomized control trial design are warranted.

\section{ACKNOWLEDGEMENTS}

The authors thank the participants in this study, as well as Mr. Bill Purves for his English editorial advice. No commercial party having a direct financial interest in the research findings reported here has conferred or will confer a benefit on the authors or on any organization with which the authors are associated. 


\section{REFERENCES}

1) Spirduso WW: Coordination and skills in complex movements. In: Physical Dimensions of Aging. Champaign: Human Kinetics, 1995 , pp $215-245$.

2) Spirduso WW, Francis KL, MacRae PG: Motor coordination and control. In: Physical Dimensions of Aging, 2nd ed. Champaign: Human Kinetics, 2005, pp 177-207.

3) Williams HG: Aging and eye-hand coordination. In: Bard C, Fleury M, Hay L (eds.), Development of Eye-Hand Coordination across the Life Span. Columbia: University of South Carolina Press, 1990, pp 327-357.

4) Guan J, Wade MG: The effect of aging on adaptive eye-hand coordination. J Gerontol B Psychol Sci Soc Sci, 2000, 55: 151-162. [Medline] [CrossRef]

5) Boisseau E, Scherzer P, Cohen H: Eye-hand coordination in aging and in Parkinson's disease. Neuropsychol Dev Cogn B Aging Neuropsychol Cogn, 2002, 9 : 266-275. [CrossRef]

6) Cheng ST, Chan AC, Yu EC: An exploratory study of the effect of mahjong on the cognitive functioning of persons with dementia. Int J Geriatr Psychiatry, 2006, 21: 611-617. [Medline] [CrossRef]

7) Ross CE, Zhang W: Education and psychological distress among older Chinese. J Aging Health, 2008, 20: 273-289. [Medline] [CrossRef]

8) Kwok JC, Hui-Chan CW, Tsang WW: Effects of aging and Tai Chi on finger-pointing toward stationary and moving visual targets. Arch Phys Med Rehabil, 2010, 91: 149-155. [Medline] [CrossRef]

9) Chiu HF, Lee HC, Chung WS, et al.: Reliability and validity of the Cantonese version of Mini-Mental Status Examination: a preliminary study. J Hong Kong Coll Psychiatr, 1994, 4: 25-28.

10) Folstein MF, Folstein SE, McHugh PR: "Mini-mental state". A practical method for grading the cognitive state of patients for the clinician. J Psychiatr Res, 1975, 12: 189-198. [Medline] [CrossRef]

11) Woods RL, Tregear SJ, Mitchell RA: Screening for ophthalmic disease in older subjects using visual acuity and contrast sensitivity. Ophthalmology, 1998, 105: 2318-2326. [Medline] [CrossRef]

12) Gyllensten AL, Hui-Chan CW, Tsang WW: Stability limits, single-leg jump, and body awareness in older Tai Chi practitioners. Arch Phys Med Rehabil, 2010, 91: 215-220. [Medline] [CrossRef]

13) Tsang WW, Hui-Chan CW: Standing balance after vestibular stimulation in Tai Chi-practicing and nonpracticing healthy older adults. Arch Phys Med Rehabil, 2006, 87: 546-553. [Medline] [CrossRef]

14) Cram JR, Kasman GS: Introduction to Surface Electromyography. Gaithersburg: Aspen Publications, 1998.

15) Fitts PM: The information capacity of the human motor system in controlling the amplitude of movement. 1954. J Exp Psychol Gen, 1992, 121: 262-269. [Medline] [CrossRef]

16) Tsang WW, Hui-Chan CW: Effects of tai chi on joint proprioception and stability limits in elderly subjects. Med Sci Sports Exerc, 2003, 35: 1962-1971. [Medline] [CrossRef]

17) Tsang WW, Hui-Chan CW: Effects of exercise on joint sense and balance in elderly men: Tai Chi versus golf. Med Sci Sports Exerc, 2004, 36: 658-667. [Medline] [CrossRef]

18) Gottsdanker R: Age and simple reaction time. J Gerontol, 1982, 37: 342-348. [Medline] [CrossRef]

19) Renaud M, Bherer L, Maquestiaux F: A high level of physical fitness is associated with more efficient response preparation in older adults. J Gerontol B Psychol Sci Soc Sci, 2010, 65B: 317-322. [Medline] [CrossRef]

20) Clark JE, Lanphear AK, Riddick CC: The effects of videogame playing on the response selection processing of elderly adults. J Gerontol, 1987 , 42 : 82-85. [Medline] [CrossRef]

21) Martini FH: The Brain and Cranial Nerves, In: Englewood Cliffs NJ (ed.), Fundamentals of Anatomy and Physiology, 3rd ed. San Francisco: International, Inc., 1995, pp 456-499.

22) Lacobie K, Lacobie C: Mahjong introduction. http://www.lacobie.org/Mahjong (Published 2005, Updated 2012).

\section{Appendix 1.}

Playing mahjong requires 4 players sitting around a square table with raised edges. A mahjong set includes 136-152 tiles (depending on the version of the game) ${ }^{6}$ ) and 3 dice. Players mix the tiles (called washing) and build up the faced-down tiles into blocks 2 layers high. They take turns to throw the dice and receive tiles according to the number thrown, sorting and arrange their own tiles into some desired spatial sequence. They then take turns drawing one tile from the face-down pile and discarding a piece ${ }^{22}$. It is important to memorize the tiles played and to anticipate other players' moves, and use these to build a strategy to maximize the chance of winning ${ }^{6}$. In completing sets, combinations of three tiles can be formed as either 3 of a kind (a pong) (one more variation is 4 of a kind) or a sequence of three numbers in a row of one suit (a chee). During playing, every player has to pay attention to certain tiles becoming available to form a pong or win the game. The player may call "pong" any time, even when it is not his turn if he has two of a kind and the latest discard makes gives him a pong. He takes the discard and reveals all three pieces ${ }^{22)}$. 